\title{
EDITORIAL
}

\section{Equity of Care among Children with heart disease in a developing country}

Pediatric cardiology is slowly evolving as a sub-speciality in Nepal. This field requires team approach, trained surgeons, interventional physicians and nursing staffs supported by modern laboratory services, equipments along with continuous professional development and research. An extensive literature documents the existence of pervasive and persistent child health, development, and health care disparities by race, ethnicity, and socioeconomic status (SES) across all domains of pediatric care. Disparities exist worldwide with regards to access to safe, effective, efficient, cost-effective and timely health care services in children. The same holds to true for children with congenital and acquired heart disease, more so in the developing countries of Asia and Africa. These variations in the standard and uniformity of care experienced during childhood can result a wide variety of health and health care outcomes, including immediate and delayed morbidity and mortality, indicating that it is crucial to identify the nature and influence of these disparities across medical and surgical interventions for congenital and adult heart disease in children. In a tertiary level cardiac referral centre of Nepal like Shahid Gangalal National Heart Centre where thousands of children across different geographic terrains, age, gender, religious, ethnic and socio-economic diversity receive care, it is important to document prospectively the nature and outcomes of care received.

Considering the large burden of infant and child mortality of Nepal, which is even dismal in children with heart disease and the relative lack of pediatric cardiac care facilities, there is an urgent need to establish new and upgrade existing facilities. The American Academy of Pediatrics also recommends that research on eliminating health and health care disparities related to race, ethnicity, and SES be a priority. Data on race, ethnicity, and SES should be collected in any research related to child cardiac health to improve their definitions and increase understanding of how these factors and their complex interrelationships affect child cardiac health and interventions. It is important to measure these variables, but it is not sufficient to use these variables alone as explanatory for differences in disease, morbidity, and outcomes without attention to the social and biologic influences they have on health throughout the life course. Besides strengthening the resources and uplifting the infrastructure of Pediatric Cardiac Care Units, undertaking research that aims to find ways to deliver optimal care to every child within economic constraints, and innovative solutions to unique geo-regional health issues in given socioeconomic millieu is one important step to move forward.

\section{Manish Shrestha}

\section{Editor}

